\title{
Our darkest hours (being neurosurgeons during the COVID-19 war)
}

\author{
Stefano Borsa ${ }^{1} \cdot$ Giulio Bertani $^{1}$ - Mauro Pluderi ${ }^{1}$ - Marco Locatelli ${ }^{1}$
}

Received: 2 April 2020 / Accepted: 7 April 2020 /Published online: 14 April 2020

(C) Springer-Verlag GmbH Austria, part of Springer Nature 2020

\section{Dear editor,}

Italy is currently facing its darkest period since World War II; as of April 1st, 2020, there are 77,635 COVID-19+ patients, 12,428 deaths, and 15,729 recovered; our country is the second most affected nation in the world by the epidemic and the first in term of victims; moreover, official reports probably underestimate COVID-19 diffusion at large scale ("we estimate there are orders of magnitude fewer infections detected ... than true infections, mostly likely due to mild and asymptomatic infections as well as limited testing capacity. In Italy, our results suggest that, cumulatively, 5.9 [1.9-15.2] million people have been infected as of March 28th, 2020, giving an attack rate of $9.8 \%$ [3.2\%-25\%] of the population" from Estimating the number of infections and the impact of nonpharmaceutical interventions on COVID-19 in 11 European countries, on behalf of the Imperial College COVID-19 Response Team, March 30th 2020 - Imperial College London).

Lombardy, our region that is the most populated in Italy (around 10.000.000), is the epicenter of the disease, and its health system is under enormous pressure trying to provide adequate care to patients presenting in our hospitals. Our region responded to the emergency, increasing the ICU beds from 900 to 1260 and converting entire hospitals to the COVID-19 + patients care; furthermore, since March 8th 2020, the regional medical system has been reorganized with the identification of specific hospitals acting as hubs for specific urgent pathologies.

As far as neurosurgery is concerned, the Regional Government assigned the hub status to three hospitals (one in Milan for the center and south of the region, one in Brescia for the east, and one in Varese for the west and north),

This article is part of the Topical Collection on Neurosurgery general

Stefano Borsa

stefano.borsa@policlinico.mi.it

1 Department of Neurosurgery, Fondazione IRCCS Cà Granda Ospedale Maggiore Policlinico, Milan, Italy where the emergencies (traumatic and vascular) are centralized; one further center (always in Milan) is a hub for the neuro-oncological cases.

On papers, this organization should have met the needs for neurosurgical treatments throughout the entire region, to the facts, it is facing the dramatic reduction of the amount of traumas registered as obvious consequence of the lockdown that paralyzed the entire country; furthermore, the emergency system is flooded by an unprecedented number of patients who need care at the same time with consequent delays in treatment of other pathologies that are not SARS; another factor to take into account is the general perception of the emergency wards as possible places of infection that leads patients, even with important neurological, cardiac, or abdominal symptoms, to avoid them.

Our university hospital has been chosen as base of the regional crisis unit, in order to coordinate the COVID-19 reorganization in the city of Milan, and has increased the ICU beds from 44 to 77; it will soon open and manage (with its medical staff) a new 200-bed hospital in the pavilions of the old Milan fair, and it has been almost entirely dedicated to the COVID-19 + patients care; we are receiving patients from the areas of Lombardy mostly affected by the disease (the provinces of Bergamo, Lodi, and Brescia); our emergency department was almost entirely dedicated to patients with SARSCov-2 with a dramatic and logic drop of other pathologies including trauma and $\mathrm{SAH}$.

In this situation, our neurosurgical activity has dropped to less than $30 \%$ of usual workload, and as medical doctors, we faced the dilemma of denying the reality and being idle or actively participating in the COVID-19 +-patient care. Some of us chose the second option and, on a voluntary basis, decided to be involved directly in a medium intensity, 31 beds, COVID-19 + ward.

After a short training with tutorials and books on acute respiratory failure, thanks to few, to tell the truth, memories of our general medicine training and with the major contribution of our internal medicine colleagues, we are now fully involved in the care of patients with COVID-19. 
Regardless of the weekends (that are considered as normal working days, due to the high volume of patients in the ward requiring continuous medical assistance and the elevated turnover of new patients), we are organized in three shifts (8.00 AM-2.00 PM, 2.00 PM-8.00 PM, and 8.00 PM-8.00 AM) with two medical doctors per shift, one in the COVID + area, defined as the "red zone" that requires specific individual protections against the virus and one in the COVID - area, the "green zone"; usually, the two specialists exchange between red and green zone every $3-4 \mathrm{~h}$ during the day and every $6 \mathrm{~h}$ during the night.

In our ward, we treat patients with COVID-19 that require $\mathrm{O} 2$ and medical therapy and we have learned to deal with venturi masks, C-PAPs, V/P ratios, HGAs, and $\mathrm{O} 2$ saturations; it could seem a one small step for an internal medicine doctor, but I can assure that it is a giant leap for a neurosurgeon. As regard for the future, given the not negligible possibility of new viral outbreaks, we neurosurgeons should reconsider our training and foresee updated didactics of internal and intensive medicine; the necessity of backup for our colleagues in the COVID wards will eventually become substantial in the days and months to come.

We hope that this tsunami, that is hitting our shores hard, will soon be no more than a memory and that we will come back to treat our patients as neurosurgeons; in the meanwhile, we do not want to regret doing nothing in our darkest hours.

Publisher's note Springer Nature remains neutral with regard to jurisdictional claims in published maps and institutional affiliations. 